\title{
To Intubate or Not to Intubate: That is the Question
}

\author{
George L. Foltin, MD, FAAP, FACEP
}

Bellevue Hospital Center, New York University Medical Center, New York, NY, USA

Progress in improving the pediatric component of emergency medical services (EMS) systems clearly is evident. Just nine years ago, Seidel noted the inadequacy of pediatric training, equipment, and system development. ${ }^{1,2}$ Since then, a total of $\$ 30$ million has been appropriated by the United States Congress for EMS for children (EMS-C) programs. That has resulted in the funding of major projects in 31 states, five targeted issues grants, and the establishment of two national resource centers. Many EMS systems nationwide-with and without federal grant assistance-have made substantial improvements in their pediatric capabilities including training protocols, triage, and quality management. ${ }^{3}$

The national survey on pediatric endotracheal intubation (PETI) by Stratton et al, ${ }^{4}$ published in the last issue of Prehospital and Disaster Medicine (V8,4,1993), reveals that $100 \%$ of state EMS agencies support training in PETI and 97\% of responding training programs provide PETI training. This is compelling evidence for progress in EMS system development and pediatric prehospital care.

Despite such optimistic findings, much work remains. Many EMS programs still lack the education, equipment, protocols, and policies that address the care of children, especially in rural areas. 5,6 The contrast between the haves and have-nots regarding EMS-C seems especially evident to the providers. In many systems, prehospital providers are clamoring for more "pediatrics," but physicians are not stepping forward to fill the void. ${ }^{7}$

A more insidious barrier to prehospital pediatric care is the intellectual argument that confirmable research must be done before adopting any intervention. Pediatric EMS is coming of age in the era of technology assessment and outcome studies. This clearly is good and appropriate, but we must avoid causing harm by denying common-sense therapies because they have not been proven efficacious in a randomized clinical trial.

The common-sense therapies of most interest in pediatric prehospital care are airway control and vascular access. Based on the epidemiology of emergency pediatric illness and injuries (e.g., trauma, near-drowning, asthma, sudden infant death syndrome [SIDS], toxic ingestions, respiratory infections, and status epilepticus) and the anatomy and pathophysiology of airway compromise, respiratory failure, cardiovascular collapse, and shock, it is reasonable to assume that endotracheal intubation is to the pediatric patient what defibrillation is to the adult.

In the survey report, the authors' voice concern that PETI is being adopted even though it has not been studied in a manner that conclusively proves it improves outcome. A review of the literature demonstrates that current success rates of PETI are lower and complication rates higher than for adult intubation, that the younger the child, the less likely PETI will be successful, and that paramedics are anxious about this procedure. Furthermore, in several studies endotracheal intubation failed to improve outcome in children who were pulseless and apneic. ${ }^{8-12}$

Addressing the outcome issue first, previous studies suffer from considerable methodological weakness. The studies have small sample sizes. More troublesome is that these studies mainly focus on the child who is pulseless and apneic. These children are predominantly asystolic and have a dismal outcome regardless of any intervention. Of note is the fact that these studies demonstrate that paramedics are capable of performing successful PETI. 8,9,12

The appropriate group to study in the emergency pediatric population is the pre-arrest patients for whom in-hospital airway management has demonstrated an 
improvement in outcome, i.e., children who suffer a primary respiratory arrest and can be resuscitated prior to prolonged anoxia. ${ }^{14-16}$ Quan et al reviewed their experience with pediatric near-drowning victims who received aggressive prehospital ALS, including PETI, in a wellorganized EMS system. ${ }^{17}$ The group of children had a short immersion time and cardiopulmonary arrest and had a remarkable $32 \%$ survival to discharge rate. Those not in arrest had a survival rate of over $60 \%$.

Although Quan's study was not a prospective analysis and it lacked a comparison group, its work makes a compelling argument for the role of prehospital intubation in improving outcome for near-drowning. The results strongly suggest that airway management makes a difference in the hypoxemic child. But, should this work alone induce all EMS systems to include PETI in their treatment armamentarium until definitive studies can be performed?

The answer is, yes, for the following reasons:

1) While there is some element of clinical equipose on this intervention (i.e., reasonable experts do not agree if PETI should be a mandatory paramedic skill), the new American Heart Association (AHA) system for evaluating the efficacy of therapies, PETI falls into classification 11a: “...a therapeutic option that is acceptable, is of uncertain efficacy and may be controversial...but is probably helpful." 18 Would those proposing to wait on definitive studies likewise suspend adult intubation on the same grounds?

2) If we believe prehospital personnel are hesitant to attempt PETI and that affects success rates, then we need to develop innovative training strategies and experience instead of abandoning something that probably works. Pediatric calls can be extremely stressful for prehospital personnel, and they make up a large number of the cases that require stress debriefing. Many a medic's career has ended from their inability to intervene to save a child's life. Addressing the anxiety and attitudinal barriers along with teaching the skill can empower the medic and help them to maintain their own emotional well-being.

3) Hypoxemic children make up the majority of chitdren for whom EMS can make a difference. Focusing on the low number of actual pediatric intubations is looking at the wrong denominator.

The paper by Stratton et al certainly suggest that arguments against PETI are falling by the wayside. By all means, we should study this important intervention. But the failure to consider a common-sense intervention for lack of conclusive data is dangerous and not the same as rejecting a therapy based on appropriate studies. Let's not throw out the baby with the bathwater.

\section{References}

1. Seidel JS, Hornbein M, Yoshiyama K, et al: Emergency medical services and the pediatric patient: Are the needs being met? Pediatrics 1984;73:769-772.

2. Seidel JS: Emergency medical services and the pediatric patient: Are the needs being met? Il. Training and equipping emergency medical services providers for pediatric emergencies. Pediatrics 1986;78:808-812.

3. Foltin G, Fuchs S: Advances in pediatric emergency medical services systems. Emerg Med Clin N Amer 1991;9:459-474.

4. Stratton SJ, Underwood LA, Whalen SM, et al: Prehospital pediatric endotracheal intubation: A survey of the United States. Prehospital and Disaster Medicine 1993:8:323-326.

5. Luten G, Foltin G (eds): Pediatric Resources for Prehospital Care Elk Grove Village, Ill.: American Academy of Pediatrics Committee of the Section on Emergency Medicine, 1990, pp 16-24.

6. Foltin G, Cooper A: Children. In Keuhl A (ed), Medical Director's Handbook. 2d ed. St. Louis: Mosby, in press.

7. Cooper A, Foltin G: Education and Training of Prehospital Personnel. In: Dieckmann RE, Planning and Managing Systems for Pediatric Emergency Care. Baltimore: Williams and Wilkins, 1992.

8. Losek JD, Halim Hennes H, Glaeser PG, et al: Prehospital care of the puiseless, nonbreathing pediatric patient. Am J Emerg Med 1987:5:370-375

9. Tsai A, Kallsen G: Epidemiology of pediatric prehospital care. Ann
Emerg Med 1987;16:284-292.

10. Losek JD, Bonadio WA, Walsh-Kelly C, et al: Prehospital pediatric endotracheal intubation performance review. Pediatric Emergency Care 1989;5:1-4.

11. Eisenberg MS, Bergner L, Hallstrom A: Cardiac resuscitation for the community: Importance of rapid provision and program planning. JAMA 1979;241:1905-1907.

12. Pointer JE, et al: Clinical characteristics of paramedic's performance of pediatric endotracheal intubation. A $m$ / Fmerg $\mathrm{Med}$ 1987;7:364-366.

13. Applebaum D: Advanced prehospital care for pediatric emergencies. Ann Emerg Med 1985;14:656.

14. Lewis JK, Minter MG, Eshelman SY, et al: Outcome of pediatric resuscitation. Ann Emerg Med 1983:297-299.

15. Nichols DG, Kettrick RG, Swedlow DB, et al: Factors influencing outcome of cardiopulmonary arrest in children. Crit Care Med 1984;12:287. Abstract.

16. Torphy DE, Minter MG, Thompson BM: Cardiorespiratory arrest and resuscitation of children. Am J Dis Child 1984;138:1099-1 102.

17. Quan L, Wentz WR, Gore EJ, et al: Outcome and predictors of outcome in pediatric submersion victims receiving prehospital care in King County, Washington. Pediatrics 1990; 86:586-593.

18. American Heart Association: Guidelines for Cardiopulmonary Resusritation and Emergency Cardiac Care. Dallas: AHA, 1992. 